\title{
Insulin Resistance and Chronic Kidney Disease in Patients with Type 1 Diabetes Mellitus
}

\author{
Mihaela Vladu, ${ }^{1,2}$ Diana Clenciu, ${ }^{2}$ Ion Cristian Efrem,, ${ }^{1,2}$ Mircea-Cătalin Forțofoiu, ${ }^{1,2}$ \\ Anca Amzolini, ${ }^{1,2}$ Simona Tudorică Micu, ${ }^{1,2}$ Maria Moţa, ${ }^{1}$ and Maria Forţofoiu ${ }^{1,2}$ \\ ${ }^{1}$ University of Medicine and Pharmacy of Craiova, Craiova, Romania \\ ${ }^{2}$ Clinical Municipal Hospital "Philanthropy" of Craiova, Craiova, Romania
}

Correspondence should be addressed to Mircea-Cătalin Forțofoiu; catalin1972@hotmail.com

Received 22 October 2016; Accepted 16 February 2017; Published 14 March 2017

Academic Editor: C. S. Johnston

Copyright (C) 2017 Mihaela Vladu et al. This is an open access article distributed under the Creative Commons Attribution License, which permits unrestricted use, distribution, and reproduction in any medium, provided the original work is properly cited.

Background and Aims. Diabetes mellitus (DM) is a chronic disease which can evolve towards devastating micro- and macrovascular complications. DM is the most frequent cause of chronic kidney disease (CKD). Insulin resistance plays an important role in the natural history of type 1 diabetes. The purpose of the study was to determine the prevalence of CKD in T1DM and the correlation with insulin resistance (IR) in patients with CKD. Materials and Methods. The study was conducted over a period of three years (2010-2013) and included patients with DM registered in the Clinical Centre of Diabetes, Nutrition and Metabolic Diseases of Dolj county. The study design was an epidemiological, transversal, noninterventional type. Finally, the study group included 200 subjects with type $1 \mathrm{DM}$. Insulin resistance (IR) was estimated by eGDR. The subjects with eGDR $\leq 7.5 \mathrm{mg} / \mathrm{kg} / \mathrm{min}$ were considered with insulin resistance. Results. CKD was found in $44 \%$ of the patients. Analyzing statistically the presence of CKD, we found highly significant differences between patients with CKD and those without CKD regarding age and sex of the patients, the duration of diabetes, glycosylated hemoglobin (HbAlc), the estimated glucose disposal rate (eGDR), and the presence of hypertension, dyslipidemia, and hyperuricaemia. In patients with CKD, age and diabetes duration are significantly higher than in those who do not have this complication. CKD is more frequent in males than in females (50.9\% men versus $34.5 \%$ women, $p=0.022)$. From the elements of metabolic syndrome, high blood pressure, hyperuricemia, and dyslipidemia are significantly increased in diabetic patients with CKD. eGDR value (expressed as $\mathrm{mg} \cdot \mathrm{kg}^{-1} \cdot \mathrm{min}^{-1}$ ) is lower in patients with CKD than in those without CKD (15.92 versus $6.42, p<0.001$ ) indicating the fact that patients with CKD show higher insulin resistance than those without CKD. Conclusions. This study has shown that insulin resistance is associated with an increased risk of CKD, but, due to the cross-sectional design, the causal relationship cannot be assessed. However, the existence of this causality and the treatment benefit of insulin resistance in type 1 diabetes are issues for further discussion.

\section{Background and Aims}

Diabetes mellitus (DM) is a chronic disease which can evolve towards devastating micro- and macrovascular complications. DM is the most frequent cause of chronic kidney disease (CKD). The diabetic chronic kidney disease (CKD) is a clinical syndrome characterized by persistent albuminuria (albumin/creatinine ratio in the spontaneous urine $\geq 30 \mathrm{mg} / \mathrm{g}$ ) and/or a sustained decline of the estimated glomerular filtration rate (eGFR) below $60 \mathrm{~mL} / \mathrm{min} / 1.72 \mathrm{~m}^{2}$. If at least one of these values is still maintained within these abnormal limits after 3 months from the first measurement, the diagnosis of diabetic CKD may be established $[1,2]$.
Insulin resistance has been linked to the pathophysiology of type 2 diabetes mellitus (T2DM) and it has been proven to play an important role in the increase of the risk of cardiovascular complications. It has been recently shown that insulin resistance plays an important role in the natural history of type 1 diabetes, although it is generally known that it is mainly due to immune destruction of the $\beta$-pancreatic cells [3].

Since 1977, Ginsberg has studied the association of insulin resistance with type 1 diabetes mellitus (T1DM) [4]. Subsequent studies $[5,6]$ showed a significant decrease in glucose disposal mediated by insulin among patients with T1DM suggesting the presence of insulin resistance. After a decade, 
this combination has aroused the interest of many research studies $[7,8]$ to investigate the possible mechanisms of insulin resistance in T1DM. Insulin resistance is a feature present in T1DM [9-11]; clamp studies on T1DM confirm its growth in patients with microalbuminuria [12] while glomerular filtration rate decreases [13]. Insulin resistance is a progressive process increasing the urinary excretion of albumin and decreasing glomerular filtration rate [14].

The purpose of the study was to determine the prevalence of CKD in T1DM and the correlation with insulin resistance (IR) in patients with CKD.

\section{Materials and Methods}

The study was conducted over a period of three years (2010-2013) and was comprised of patients with DM registered in the Clinical Centre of Diabetes, Nutrition and Metabolic Diseases of Dolj county.

The study design was an epidemiological, transversal, noninterventional type. Finally, the study group included 200 subjects with T1DM.

Anamnestic data have been analyzed (age, sex, duration of DM, history of arterial hypertension, dislipidemia, and hyperuricemia), as well as paraclinical data (urea, creatinine, uric acid, cholesterol, triglycerides, Alc, and urinary albumin-to-creatinine ratio). The estimated glomerular filtration rate (eGFR) has also been calculated according to the Modification of Diet in Renal Disease (MDRD) equation. The CKD stages have been established according to KDIGO 2012 definition. In predicting the risk for outcome of CKD, we used GFR and albuminuria category. GFR from 60 to $89 \mathrm{~mL} / \mathrm{min} / 1.73 \mathrm{~m}^{2}$ is classified as CKD stage 2, from 45 to $59 \mathrm{~mL} / \mathrm{min} / 1.73 \mathrm{~m}^{2}$ as CKD stage $3 a$, from 30 to $44 \mathrm{~mL} / \mathrm{min} / 1.73 \mathrm{~m}^{2}$ as CKD stage $3 \mathrm{~b}$, and from 15 to $29 \mathrm{~mL} / \mathrm{min} / 1.73 \mathrm{~m}^{2}$ as CKD stage 4 . Stage 1 is defined by GFR over $90 \mathrm{~mL} / \mathrm{min} / 1.73 \mathrm{~m}^{2}$ and stage 5 by GFR under $15 \mathrm{~mL} / \mathrm{min} / 1.73 \mathrm{~m}^{2}$. Albuminuria categories were $\mathrm{A} 1<30 \mathrm{mg} / \mathrm{g}$ (normally to moderately increased), A2 = 30-300 mg/g (moderately increased), and A3 > $300 \mathrm{mg} / \mathrm{g}$ (severely increased).

Insulin resistance was evaluated by the estimated glucose disposal rate (eGDR). Subjects with eGDR $\leq 7.5 \mathrm{mg} / \mathrm{kg} / \mathrm{min}$ were considered with IR. The euglycemic-hyperinsulinemic clamp is the accepted standard for measurement of insulin sensitivity; however, it is not practical for use in the clinical setting. The eGDR can be calculated using routine clinical measures: the glycosylated hemoglobin (HbAlc), the presence of hypertension, and the waist circumference $[15,16]$. We used the eGDR because it shows good correlation with IR measured by the euglycemic-hyperinsulinemic clamp and has been validated for the estimation of insulin sensitivity in individuals with type $1 \mathrm{DM}$.

2.1. Statistical Analysis. The recorded data have been analyzed using the Statistical Package for the Social Sciences (SPSS), version 17.00, software (IBM Corporation, Armonk, NY, USA). We performed analysis of the entire study population and separate statistics for each of the 3 groups. The
TABLE 1: Characteristics of the studied population.

\begin{tabular}{lc}
\hline Parameters & Value \\
\hline Sex $(\mathrm{M} / \mathrm{F})$ & $116(58 \%) / 84(42 \%)$ \\
Age & $37,57 \pm 12,45$ \\
Age at the onset of DM & $21,49 \pm 8,49$ \\
Duration of DM & $16,21 \pm 8,97$ \\
BMI $\left(\mathrm{kg} / \mathrm{m}^{2}\right)$ & $23,84 \pm 3,72$ \\
Abdominal circumference & $88,43 \pm 10,21$ \\
Hip circumference & $96,34 \pm 7,77$ \\
Waist/hip index & $0,91 \pm 0,07$ \\
eGFR & $92,39 \pm 25,17$ \\
eGDR & $10,06 \pm 5,56$ \\
Total cholesterol & $170,35 \pm 41,61$ \\
HDL-cholesterol & $61,35 \pm 57,87$ \\
Triglyceride & $114,6 \pm 66,68$ \\
Alc & $8,4 \pm 1,58$ \\
CKD & $44 \%$ \\
Hypertension & $52,5 \%$ \\
Dyslipidemia & $55,5 \%$ \\
Hyperuricemia & $31 \%$ \\
\hline
\end{tabular}

methods used were $t$-test, Mann-Whitney test, Chi-square test, and Cramer test as appropriate. We used the following interpretation of $p$ values: $p<0.05$, the difference between the two means is significant $(\mathrm{S}) ; p<0.01$, the difference between the two means is highly significant (HS); $p<0.001$, the difference between the two averages is very highly significant (VHS); $p>0.05$, the difference between the two means is not significant (NS)

\section{Results}

The study included 200 subjects with type 1 diabetes (116 men and 84 women). Table 1 summarizes the characteristics of the population studied. Data are presented as mean \pm standard deviation.

Insulin resistance was found in $41.5 \%$ of patients with type 1 DM (Figure 1). CKD was found in $44 \%$ of the patients (Figure 2). Statistically analyzing the presence of CKD, there were statistically highly significant differences between the patients with $C K D$ and those without $C K D$ with regard to age and sex of the patients, duration of diabetes, HbAlc, eGDR, and the presence of hypertension, dyslipidemia, and hyperuricaemia (Table 2).

In patients with $\mathrm{CKD}$, the age and diabetes duration are significantly higher than in those who do not have this complication. CKD is more frequent in males than in females (50.9\% men versus $34.5 \%$ women, $p=0.022$ ). High blood pressure, hyperuricemia, and dyslipidemia are significantly increased in the diabetic patients with CKD.

eGDR value (expressed as $\mathrm{mg} \cdot \mathrm{kg}^{-1} \cdot \mathrm{min}^{-1}$ ) is lower in patients with CKD than in those without CKD (15.92 versus 6.42, $p<0.001)$, indicating that patients with CKD show higher insulin resistance than those without CKD (Figure 3). 
TABLE 2: Characteristics of patients with CKD.

\begin{tabular}{lccc}
\hline Variables & With CKD & Without CKD & N/A \\
\hline Number of patients & $88(44 \%)$ & $112(56 \%)$ & $p<0,001$ \\
Age (years) & $43,78 \pm 10,57$ & $32,68 \pm 11,64$ & $p=0,022$ \\
Sex $(\mathrm{M} / \mathrm{F})$ & $59 / 29$ & $57 / 55$ & $p<0,001$ \\
Duration of DM (years) & $20,05 \pm 6,62$ & $13,2 \pm 9,44$ & $p=0,057$ \\
BMI $\left(\mathrm{kg} / \mathrm{m}^{2}\right)$ & $24,28 \pm 3,68$ & $23,49 \pm 3,73$ & $p=0,671$ \\
AC $(\mathrm{cm})$ & $88,88 \pm 10,83$ & $88,08 \pm 9,73$ & $p<0,001$ \\
Alc $(\%)$ & $8,88 \pm 1,71$ & $8,02 \pm 1,26$ & $p<0,001$ \\
eGDR $\left(\mathrm{mg} \cdot \mathrm{kg}^{-1} \cdot \mathrm{min}^{-1}\right)$ & $6,42 \pm 3,44$ & $15,92 \pm 5,22$ & $p<0,001$ \\
Dyslipidemia & $59(67 \%)$ & $37(33 \%)$ & $p<0,001$ \\
Hypertension & $61(69,3 \%)$ & $34(30,3 \%)$ & $p<0,001$ \\
Hyperuricemia & $77(87,5 \%)$ & $14(12,5 \%)$ & \\
\hline
\end{tabular}

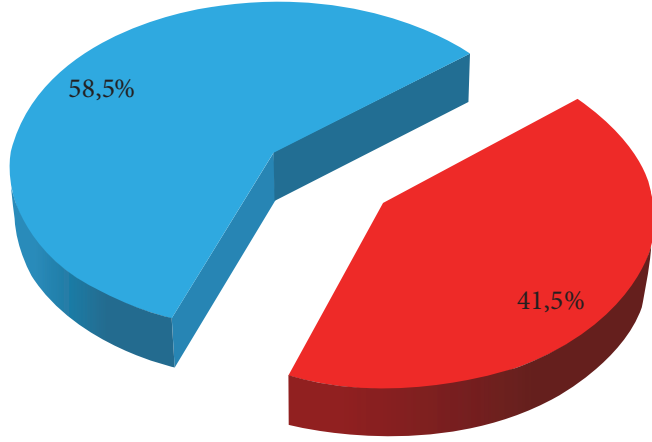

Without IR (eGDR > 7.5)

With IR $(e G D R \leq 7.5)$

FIGURE 1: Insulin resistance in type $1 \mathrm{DM}$ patients.

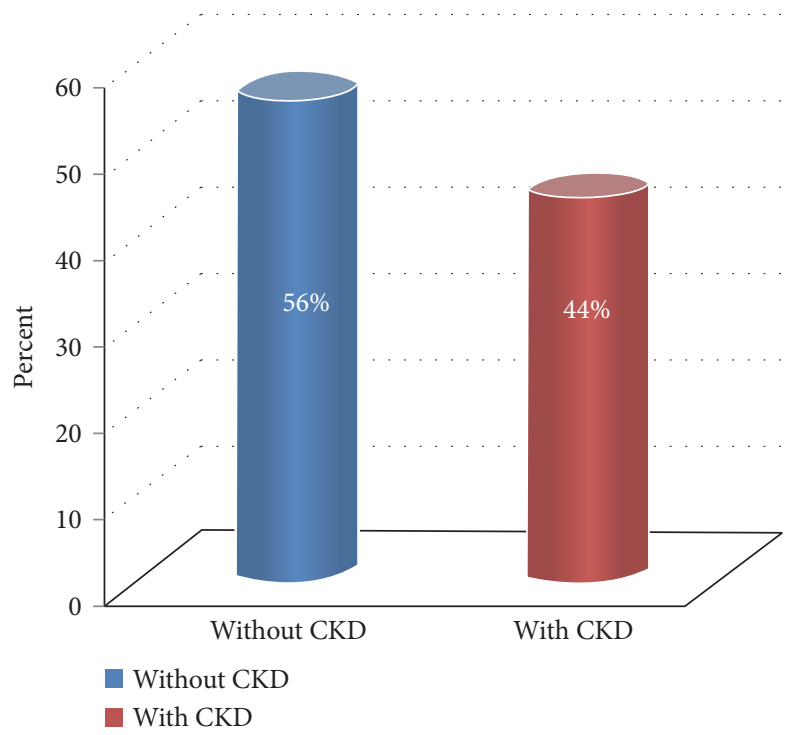

FIGURE 2: Graphic representation of CKD.

By performing the logistic regression analysis of the stepwise type where we introduced all statistically significant parameters in Table 2, we obtained the final regression model shown in Table 3.

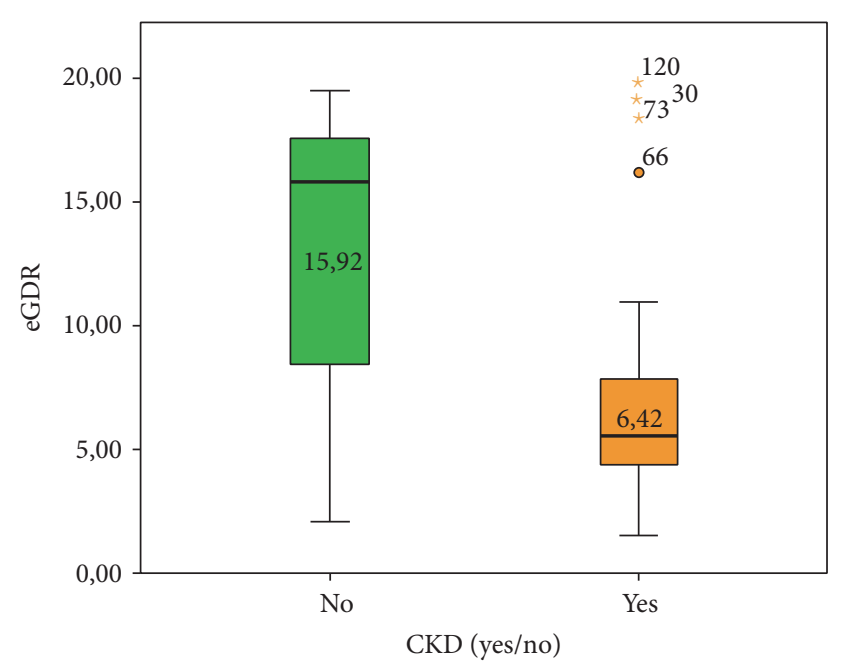

IR in patients without CKD

IR in patients with CKD

Figure 3: Insulin resistance in CKD patients.

TABLE 3: The final model of logistic regression for CKD.

\begin{tabular}{lccc}
\hline & OR & $95 \%$ CI & $p$ \\
\hline Age & 1,086 & $1,056-1,116$ & $p<0,001$ \\
Sex & 0,509 & $0,286-0,909$ & $p=0,022$ \\
Duration of DM & 1,105 & $1,062-1,150$ & $p<0,001$ \\
Alc & 1,450 & $1,186-1,774$ & $p<0,001$ \\
eGDR & 0,744 & $0,684-0,810$ & $p<0,001$ \\
Dyslipidemia & 10,714 & $5,354-21,442$ & $p<0,001$ \\
Hypertension & 12,167 & $6,099-24,269$ & $p<0,001$ \\
Hyperuricemia & 20,647 & $8,937-47,702$ & $p<0,001$ \\
\hline
\end{tabular}

Analyzing the area under the ROC curve, used to assess the usefulness of studying the parameters from Table 3 as independent predictors for the occurrence of CKD in patients with type 1 diabetes, the best predictor appears to be the eGDR value followed by the duration of diabetes, age, 
TABLE 4: Area under the ROC curve analysis for statistically significant parameters associated with CKD.

\begin{tabular}{lcc}
\hline & Area under the ROC curve & $95 \%$ CI \\
\hline eGDR & $\mathbf{0 , 8 2 9}$ & $0,770-0,888$ \\
Age & $\mathbf{0 , 7 7 4}$ & $0,708-0,840$ \\
Hypertension & $\mathbf{0 , 7 7 2}$ & $0,705-0,839$ \\
Hyperuricemia & $\mathbf{0 , 7 7 1}$ & $0,701-0,841$ \\
Duration of DM & $\mathbf{0 , 7 7 0}$ & $0,704-0,836$ \\
Dyslipidemia & $\mathbf{0 , 7 5 5}$ & $0,687-0,824$ \\
Alc & 0,663 & $0,585-0,741$ \\
Sex & 0,581 & $0,501-0,660$ \\
\hline
\end{tabular}

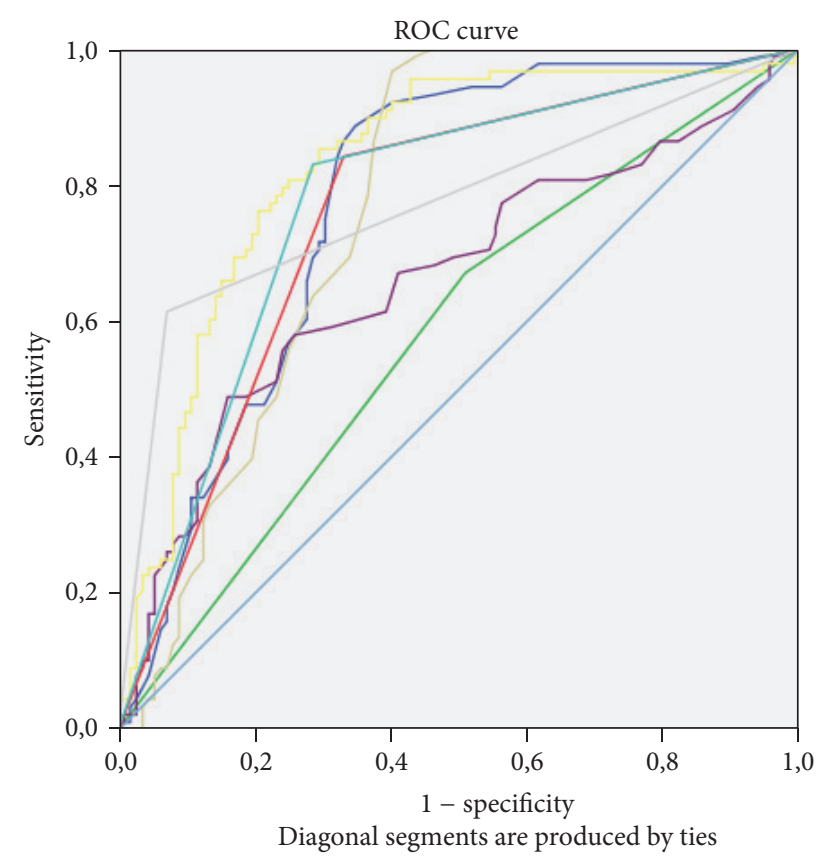

Source of the curve

$\begin{array}{ll}- \text { Age } & \text { Dyslipidemia } \\ \text { Sex } & \text { Arterial hypertension } \\ \text { DM duration } & - \text { Hyperuricemia } \\ \text { Alc } & - \text { Reference line } \\ \text { eGDR } & \end{array}$

FIgUre 4: Analysis of the area under the ROC curve for the eGDR in patients with CKD.

hypertension, dyslipidemia, and blood pressure (Table 4 and Figure 4).

\section{Discussions}

Older age and long evolution of DM are factors known and shown in numerous studies to be associated with the development and progression of chronic complications in diabetes, whereas this new concept of insulin resistance has appeared lately in type $1 \mathrm{DM}$ and it has gained ground worldwide being accepted by doctors and researchers. Its association with the emergence of chronic vascular complications in particular triggered the development of several studies that try to explain the link between insulin resistance and type 1 DM.

In European countries such as Finland, the country with the highest rate of incidence and prevalence of diabetes type 1 in the world, insulin resistance is associated with older age, longer duration of diabetes, and an increased amount of fat tissue [17] with a family history of type $2 \mathrm{DM}$, with poor glycemic control [15] and increased serum lipid levels. In Asian countries, a low prevalence of this disease prevented the researchers from investigating the link between insulin resistance and type $1 \mathrm{DM}$. In general, the prevalence of insulin resistance in the Asian population both in diabetic and in nondiabetic populations is relatively small compared to the Caucasian race, this thing being attributed to lower rates of obesity in Asians.

In this study, we evaluated the prevalence of insulin resistance in patients with type 1 diabetes using clinical score eGDR, its results showing a prevalence of $41.5 \%$, similar to other European countries where the prevalence cited in studies is $32-48 \%$.

As in the studies conducted in Europe, older age, longer duration of diabetes development, hypertension, and dyslipidemia are more frequent characteristics among adults with type $1 \mathrm{DM}$ with insulin resistance.

The small sample of 200 patients used in this study compared with the studies conducted in the European Union may explain some of the differences which appear. In addition, both groups have a large number of subjects under insulin treatment inadequately dosed due to the risk of hypoglycemia especially in the context given by the presence of CKD. The results of this study showed that insulin resistance, measured by eGDR, is more frequent in patients with CKD than in those without CKD.

\section{Conclusions}

(i) This study has shown that insulin resistance is associated with an increased risk of CKD, but, because of the cross-sectional design, the causal relationship cannot be assessed. However, the existence of this causality and the benefit of treatment for insulin resistance in type $1 \mathrm{DM}$ are issues for further discussion.

(ii) In conclusion, our study has shown that insulin resistance is a constant factor associated with CKD in type $1 \mathrm{DM}$ and it may be useful in the future for CKD screening.

\section{Conflicts of Interest}

The authors declare that they have no competing interests regarding the publication of this paper.

\section{Authors' Contributions}

All authors contributed equally to the preparation of this manuscript and thus share first authorship. 


\section{References}

[1] National Kidney Foundation, "K/DOQI clinical practice guidelines for chronic kidney disease: evaluation, classification, and stratification," American Journal of Kidney Diseases, vol. 39, no. 2, supplement 1, pp. S1-S266, 2002.

[2] KDIGO, "KDIGO 2012 guideline for evaluation and management of CKD," Kidney International Supplements, vol. 3, pp. 1962, 2013.

[3] G. M. Reaven, "Role of insulin resistance in human disease," Diabetes, vol. 37, no. 12, pp. 1595-1607, 1988.

[4] H. N. Ginsberg, "Investigation of insulin sensitivity in treated subjects with ketosis prone diabetes mellitus," Diabetes, vol. 26, no. 4 , pp. 278-283, 1977.

[5] R. A. DeFronzo, R. Hendler, and D. Simonson, "Insulin resistance is a prominent feature of insulin-dependent diabetes," Diabetes, vol. 31, no. 9, pp. 795-801, 1982.

[6] S. Del Prato, R. Nosadini, A. Tiengo et al., "Insulin-mediated glucose disposal in type I diabetes: evidence for insulin resistance," Journal of Clinical Endocrinology and Metabolism, vol. 57, no. 5, pp. 904-910, 1983.

[7] A. D. Baron, M. Laakso, G. Brechtel, and S. V. Edelman, "Mechanism of insulin resistance in insulin-dependent diabetes mellitus: a major role for reduced skeletal muscle blood flow," Journal of Clinical Endocrinology and Metabolism, vol. 73, no. 3, pp. 637-643, 1991.

[8] S. Mäkimattila, A. Virkamäki, R. Malmström, T. Utriainen, and H. Yki-Jarvinen, "Insulin resistance in type I diabetes mellitus: a major role for reduced glucose extraction," Journal of Clinical Endocrinology and Metabolism, vol. 81, no. 2, pp. 707-712, 1996.

[9] A. Uruska and A. Araszkiewicz, "Insulin resistance in patients with type 1 diabetes," Pediatric Endocrinology, Diabetes and Metabolism, vol. 15, no. 2, pp. 119-123, 2009.

[10] S. A. Dib, "Insulin resistance and metabolic syndrome in type 1 diabetes mellitus," Arquivos Brasileiros de Endocrinologia \& Metabologia, vol. 50, no. 2, pp. 250-263, 2006.

[11] K. J. Nadeau, J. G. Regensteiner, T. A. Bauer et al., "Insulin resistance in adolescents with type 1 diabetes and its relationship to cardiovascular function," Journal of Clinical Endocrinology and Metabolism, vol. 95, no. 2, pp. 513-521, 2010.

[12] R. Trevisan, D. Bruttomesso, M. Vedovato et al., "Enhanced responsiveness of blood pressure to sodium intake and to angiotensin II is associated with insulin resistance in IDDM patients with microalbuminuria," Diabetes, vol. 47, no. 8, pp. 1347-1353, 1998.

[13] M. Svensson, Z.-W. Yu, and J. W. Eriksson, "A small reduction in glomerular filtration is accompanied by insulin resistance in type I diabetes patients with diabetic nephrophathy," European Journal of Clinical Investigation, vol. 32, no. 2, pp. 100-109, 2002.

[14] L. M. Thorn, C. Forsblom, J. Fagerudd et al., "Metabolic syndrome in type 1 diabetes: association with diabetic nephropathy and glycemic control (the FinnDiane study)," Diabetes Care, vol. 28, no. 8, pp. 2019-2024, 2005.

[15] K. V. Williams, J. R. Erbey, D. Becker, S. Arslanian, and T. J. Orchard, "Can clinical factors estimate insulin resistance in type 1 diabetes?" Diabetes, vol. 49, no. 4, pp. 626-632, 2000.

[16] J. J. Chillarón, A. Goday, J. A. Flores-Le-Roux et al., "Estimated glucose disposal rate in assessment of the metabolic syndrome and microvascular complications in patients with type 1 diabetes," The Journal of Clinical Endocrinology \& Metabolism, vol. 94, no. 9, pp. 3530-3534, 2009.
[17] G. Pambianco, T. Costacou, and T. J. Orchard, "The prediction of major outcomes of typel diabetes: a 12-year prospective evaluation of three separate definitions of the metabolic syndrome and their components and estimated glucose disposal rate: the Pittsburgh Epidemiology of Diabetes Complications Study experience," Diabetes Care, vol. 30, no. 5, pp. 1248-1254, 2007. 


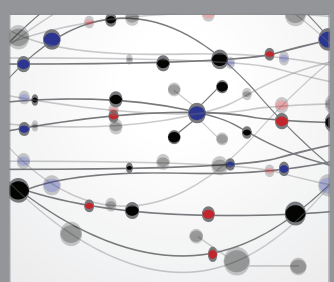

The Scientific World Journal
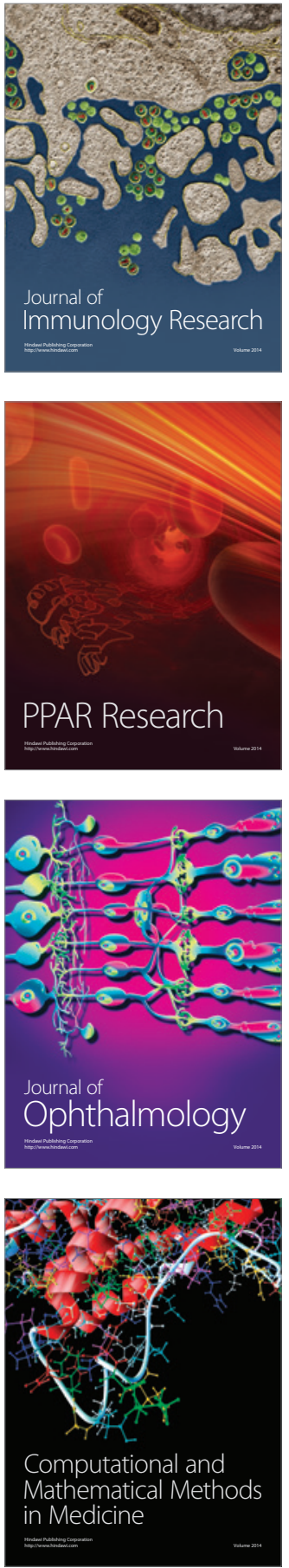

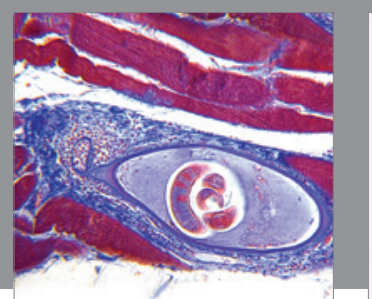

Gastroenterology Research and Practice
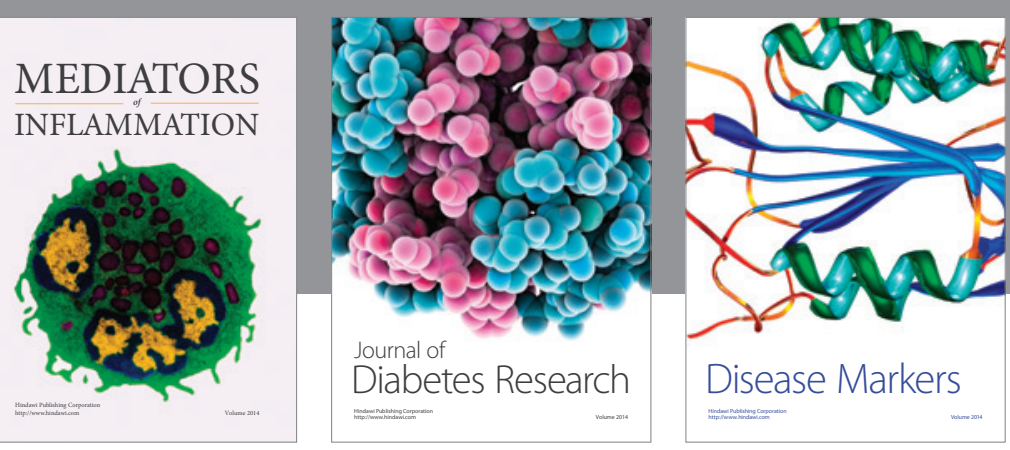

Disease Markers

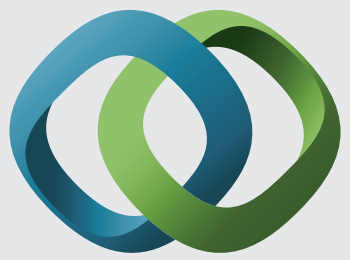

\section{Hindawi}

Submit your manuscripts at

https://www.hindawi.com
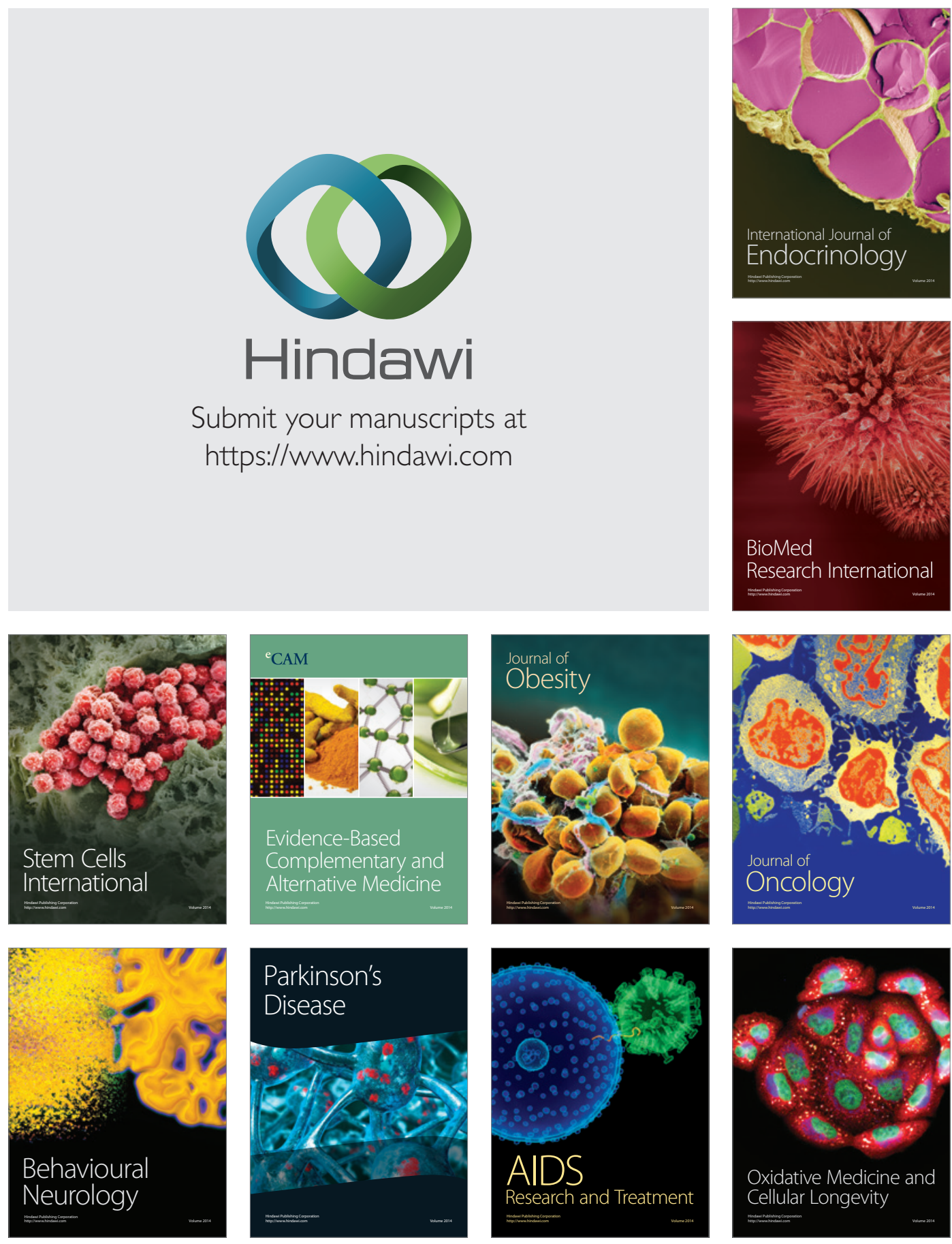